\title{
Complemento demográfico por matemidad en el marco de las políticas de igualdad. Análisis de objetivos, resultados y consecuencias
}

\author{
Rafael Granell Pérez \\ Concha Salvador Cifre
}

RESUMEN: Este trabajo analiza, desde la perspectiva de la Economía Pública, el Complemento Demográfico por Maternidad introducido en el Sistema de Seguridad Social Español en 2016. La medida supone una mejora de las pensiones contributivas de jubilación, viudedad e incapacidad permanente percibidas por madres de al menos dos hijos, con la pretensión de reducir las diferencias de género y reconocer la maternidad como aportación al sistema de pensiones. Se examinan ambos objetivos y los puntos críticos de esta medida de política pública, incidiendo en sus efectos distributivos: el impacto de la medida en el sistema de pensiones, las diferencias de trato que genera su aplicación y sus efectos sobre la brecha de género y sobre la desigualdad entre las mujeres. Para estimar los principales resultados se han utilizado los microdatos de la Muestra Continua de Vidas Laborales y datos agregados facilitados por el Ministerio de Empleo y Seguridad Social. Esta medida ha tenido unos resultados destacables, beneficiando al $58 \%$ de las nuevas pensionistas, pero también pueden subrayarse aspectos críticos, como la discriminación para algunos colectivos de mujeres y hombres, su modesto efecto sobre la reducción de la brecha de género o su impacto sobre la desigualdad entre las mujeres. Se finaliza planteando otras políticas de igualdad de género, referidas al sistema de pensiones, que podrían mejorar estos resultados.

PALABRAS CLAVE: Seguridad Social, pensiones, brecha de género, desigualdad.

CLAVES ECONLIT: H89, H55, J16, J26.

Cómo citar este artículo / How to cite this article: GRANELL, R. \& SALVADOR, C. (2020): "Complemento demográfico por maternidad en el marco de las políticas de igualdad. Análisis de objetivos, resultados y consecuencias", CIRIEC-España, Revista de Economía Pública, Social y Cooperativa, 98, 287-322. DOI: 107203/ CIRIEC-E.98.13570.

Correspondencia: Rafael Granell Pérez, profesor Titular de Universidad de la Universitat de València, Rafael.Granell@uv.es; Concha Salvador Cifre, profesora Titular de Universidad de la Universitat de València, Concha.Salvador@uv.es. 


\section{EXPANDED ABSTRACT}

\section{Demographic supplement for motherhood within the framework of equality policies. Analysis of objectives, results and outcomes}

This study analyses, from the perspective of public economics, the Demographic Supplement for Motherhood (CDM) introduced in the Spanish Social Security System in 2016. This measure is a supplement added to the contributory pension received on retirement, widowhood or disability for women who began to receive a pension and who have had two or more children. It was introduced with two objectives in mind: to reduce the gender gap in pensions and to socially recognise the contribution of motherhood to the pension system.

In this paper, a socioeconomic analysis of this measure is carried out. The objectives and issues of this measure are reviewed and its distributional effects are analysed. The main source of information are data provided by the Ministry of Employment and Social Security, in particular the Continuous Sample of Working History (MCVL).

This is a new approach, with the focus being on public economics rather than on legal aspects and use of the MCVL data which, for the first time, includes measurements related to the CDM.

The main objectives of this study are: 1) to estimate the impact that the CDM has had on the pension system during the first year of application; 2) to analyse the differences in treatment it generates; and, 3) to assess its distributional effects on the gender gap in pensions and on inequality among women. The study finishes with an analysis of other equality policies and puts forward the main conclusions reached and proposals for alternative measures.

The results show that the CDM has affected $58.4 \%$ of new female pensioners. The women who have benefitted most from this measure are those who receive a widow's pension (50.9\%), followed by retirement pension (39.8\%) and disability pension (9.2\%). Their sociodemographic characteristics show that $53.4 \%$ of the supplement is paid to mothers with 2 children, while women not receiving CDM tend to have higher educational levels than those who are in receipt of the supplement. It also shows that self-employed workers tend to have more than one child and, therefore, receive the CDM in a greater proportion (62.5\%) than employed workers $(57.5 \%)$. The estimated cost of this measure amounted to 64 million euros in 2016 (approximately $0.05 \%$ of the expenditure on contributory pensions), a figure that will increase considerably as the supplement is extended to future female pensioners.

Among the main criticisms highlighted by this study is the difference in treatment this measure generates. It purports to be a measure which acknowledges the value of motherhood yet it discriminates 
against many mothers by excluding women who were pensioners before 2016 and who are the most affected, historically, by more unfavourable family and work structures. In addition, other categories excluded are mothers with only one child, women who took voluntary retirement, women who receive non-contributory pensions and women who do not receive any pension. Finally, the measure works against the principle of equality between men and women, promulgated by the European Union, because it discriminates against fathers.

Another criticism is that the amount of the CDM increases as the pension rises and continues to be paid even when the maximum pension is reached. This means that those women with higher pensions benefit more from the supplement, which is contrary to measures applied in other countries. One suggestion to improve the equality of this measure is that the supplement should have an upper limit.

Furthermore, its effect on reducing inequality has been insignificant. As a measure of equality between men and women, the CDM has reduced the gender gap between new pensioners $(2.2 \%)$ but its effect on the whole system is very poor $(0.22 \%)$. The pension gap between men and women in Spain is still very wide at a rate of $29.3 \%$ and much more work needs to be done to reduce this gap.

Finally, a pension system that gives women greater rights for raising children can reinforce traditional roles, discouraging mothers from entering the formal labour market and fathers from taking a break from their professional careers. This is why these measures are being questioned by the Court of Justice of the European Union in terms of equal treatment between mothers and fathers.

The CDM does not address the causes of the problem of gender discrimination. It does not address discrimination in employment nor does it offer the support required in the workplace for reconciling maternity and paternity leave. It is ineffective as a stimulus to change labour behaviour of women because its effect is in the long term and it is an outdated measure that can be counterproductive.

In light of these problems, the priority for the government would be to establish authentic equality policies which create opportunities for both men and women to develop their full potential. 1) labour market policies that eliminate gender differences (access to employment, wages and job promotion at work), improving the flexibility and the rationale of the working day, and 2) reconciliation policies related to family and work life, aimed at all workers, that recognise fathers and mothers as having co-responsibility for childcare.

The following measures are proposed to reduce the gender gap in pensions:

a) Replace the current CDM with an additional contribution period per child or a fixed amount supplement, in recognition of the period of time dedicated to bringing up children. 
If contribution years were added for accessing the pension, the number of women with contributory pensions would increase. To avoid the same negative aspects already highlighted, it should be implemented in a way that covers all kind of pensions and gives greater support to mothers of children with disabilities.

b) Improve non-contributory (universal) pensions, which are those of lesser value. This measure would increase the number of women with pensions in their own right, thereby reducing the coverage gap and the pension gap of the total population (including non-pensioners). This extension is vital to stop the pension system being a welfare system (low coverage) and being outdated and out of touch (excessively linked to family relationships).

Our further studies will be centred on these proposals with data provided by the MCVL in future years.

KEYWORDS: Social Security, pensions, gender gap, inequality. 


\section{Introducción'}

La igualdad entre mujeres y hombres es un principio jurídico universal sobre derechos humanos reconocido en diversos textos internacionales, entre los que destaca la Carta de las Naciones Unidas (1945), la Declaración Universal de los derechos Humanos (1948) y la Convención sobre la eliminación de todas las formas de discriminación contra la mujer (1979), aprobados por la Asamblea General de Naciones Unidas. Al mismo tiempo, la legislación de la Unión Europea (UE) exige la igualdad de género en el empleo y en materia de seguridad social, aunque permite determinadas excepciones justificadas de carácter transitorio (Carta de los Derechos Fundamentales de la UE, 2000, art. 23; Directiva 2004/113/CE, arts. 4 y 6), entre ellas, las mejoras en la pensión por criar a los hijos (Directiva 79/7/CE, art. 7) o la compensación de las desventajas en las carreras profesionales (Tratado de Funcionamiento de la UE, 2010, art. 157.4).

La Constitución Española (arts. 9.2 y 14) proclama el derecho a la igualdad y a la no discriminación por razón de sexo y la obligación de los poderes públicos de promover las condiciones para su cumplimiento. Así se reconoce en la Ley Orgánica 3/2007, de 22 de marzo, para la igualdad efectiva de mujeres y hombres (Ley de lgualdad), junto con la necesidad de acciones positivas dirigidas a combatir la desigualdad derivada de la discriminación salarial, en el empleo y en el sistema de pensiones, eliminando los obstáculos y estereotipos sociales que impiden reducirla, fomentando, entre otras acciones, el derecho a la conciliación y la corresponsabilidad en obligaciones familiares.

El Complemento Demográfico por Maternidad (CDM) es la principal apuesta del Gobierno español como acción positiva por mejorar las pensiones de las mujeres, en el marco del "Plan integral de apoyo a la familia 2015-2017" (Consejo de Ministros, 2015), siendo su pretensión dar cumplimiento a la Recomendación $17^{\mathrm{a}}$ del Informe del Pacto de Toledo y a las recomendaciones de la UE en materia de reducción de la brecha de género en pensiones (Comisión de Seguimiento y Evaluación del Pacto de Toledo, CSEPT, 2015a).

Esta medida complementa determinadas prestaciones contributivas del sistema de Seguridad Social y del Régimen de Clases Pasivas del Estado, causadas desde el inicio de 2016, teniendo derecho al complemento las mujeres que hayan tenido al menos dos hijos a lo largo de su vida (CCOO, 2016). Las principales condiciones para su aplicación son las siguientes (Ley General de Seguridad Social, art. 60 y Ley de Clases Pasivas del Estado, D.A. 18 ):

1.- El presente trabajo ha recibido el apoyo institucional del Proyecto HIECPU/2019/2 de la Conselleria de Hacienda y Modelo Económico de la Generalitat Valenciana. 
- Se aplica a las pensiones de jubilación, viudedad e incapacidad permanente, salvo a jubilaciones anticipadas voluntarias y a funcionarias jubiladas antes de la edad forzosa. En caso de acceder a la jubilación parcial, el complemento puede percibirse desde el acceso a la jubilación plena.

- El importe del complemento es el resultado de aplicar un porcentaje de incremento sobre la cuantía inicial de la pensión, en función del número de hijos nacidos 0 adoptados hasta ese momento. Es el $5 \%, 10 \%$ y $15 \%$, en caso de 2 hijos, 3 hijos y 4 o más hijos, respectivamente.

- También se establecen las siguientes condiciones particulares:

- Si la pensión inicial alcanza o supera la pensión máxima, el porcentaje se aplicará a la pensión máxima y el complemento será el 50\% del importe resultante.

- Si la pensión fuese inferior a la pensión máxima, pero la superase al sumar el complemento por maternidad, entonces el complemento será el $50 \%$ de este exceso.

- Si se tiene derecho a varias pensiones, el complemento se aplicará solo a la de jubilación o, en su defecto, a la de mayor cuantía y se estimará sobre la pensión inicialmente causada si se cobra el complemento a mínimos.

En cuanto al objetivo de esta medida, según explican sus artífices, es doble (CSEPT, 2015a). Por un lado, la reducción de la brecha de género en las pensiones, derivada de la doble penalización de las trabajadoras que optan por la maternidad. Y, por otro lado, el reconocimiento social de la maternidad por su contribución real al sistema de pensiones, considerando la maternidad como cotización demográfica, utilizando un criterio de contributividad ampliada (Ministerio de Empleo y Seguridad Social, 2015). Se trata, de compensar a las mujeres (CSEPT, 2015a: 2, 8, 27) "su esfuerzo por haber tenido hijos... reconocer por el sistema de Seguridad Social una aportación, los hijos, como elemento clave en la sostenibilidad futura del sistema", pero no cualquier esfuerzo, puesto que solo se favorece a "quien ha hecho el doble esfuerzo de trabajar y tener hijos" y a quienes "han hecho el esfuerzo por encima de la media".

La maternidad explica gran parte de la brecha salarial (Kleven et al., 2018), puesto que las mujeres dedican más tiempo a la crianza de los hijos (Lázaro et al., 2004), de ahí que se defienda la necesidad de políticas públicas para la reducción de la brecha de género y para que no disminuya la tasa de natalidad. En este contexto nos preguntamos, el pretendido reconocimiento social de la maternidad del complemento, ¿contribuye a solucionar los problemas demográficos o no es más que una medalla con valor monetario? Los resultados de medidas puntuales como el CDM, que actúan sobre las consecuencias sin considerar las causas, puede que no sean suficientes.

Además, el CDM, como acción positiva, supone una excepción al principio de igualdad de género y puede ser potencialmente contraproducente, de manera que su aplicación solo está justificada si se vela por minimizar estas consecuencias negativas y es una medida de carácter transitorio. Esta visión crítica de las acciones positivas prioriza las medidas en pro de la igualdad en el empleo, en los salarios y en el reparto de las responsabilidades domésticas. No obstante, de alguna manera hay que 
actuar para reducir las diferencias actuales, siendo importante que las políticas transitorias no discriminen y estimulen el comportamiento de la corresponsabilidad familiar, para progresar en términos de igualdad real tanto en el ámbito del mercado de trabajo como en el ámbito familiar.

El CDM es un tema controvertido, que hasta el momento se ha analizado desde una perspectiva fundamentalmente jurídica. Nuestra intención en este trabajo es realizar un análisis socioeconómico de esta medida, sistematizando los estudios ya publicados y extrayendo resultados de la información proporcionada por el Ministerio de Empleo y Seguridad Social, en especial de la Muestra Continua de Vidas Laborales.

Se trata de un análisis novedoso porque su enfoque es el de la Economía Púbica, frente a los análisis jurídicos anteriores, y porque las estimaciones se realizan principalmente sobre los datos de la MCVL-2016, que contiene por primera vez las principales magnitudes del complemento.

El artículo se estructura de la siguiente forma. Comenzamos exponiendo las fuentes y la metodología utilizadas, para desarrollar seguidamente los tres principales objetivos del trabajo. En primer lugar, la estimación del impacto que ha tenido la medida en el sistema de pensiones en su primer año de vigencia (2016); en segundo lugar, el análisis de las diferencias de trato que genera su aplicación; y, por último, la valoración de sus efectos distributivos, distinguiendo entre su efecto sobre la brecha de género en las pensiones y sobre la desigualdad entre las mujeres. Finalizamos el trabajo con un análisis de las políticas de igualdad y con las principales conclusiones obtenidas, acompañadas de una propuesta de medidas alternativas.

\section{Fuentes y metodología}

Para la evaluación del CDM llevaremos a cabo un análisis descriptivo, realizando valoraciones de carácter cualitativo y cuantitativo, utilizando los datos publicados por el Ministerio de Empleo y Seguridad Social (en adelante, MESS), incluyendo la Muestra Continua de Vidas Laborales correspondiente a 2016 (en adelante, MCVL-2016).

Esta muestra es representativa del colectivo de personas que reciben prestaciones contributivas por parte de la Seguridad Social y no se realiza mediante una encuesta, sino que se basa en los registros administrativos de las personas que tienen una relación con la Seguridad Social. Ofrece información muy detallada sobre cuatro grandes apartados: datos demográficos (edad, género, nacionalidad o nivel educativo); datos de afiliación (régimen y grupo de cotización, actividad económica, tipo de contrato, etc.); bases de cotización (año de cotización y bases anual y mensual); y pensiones 
(tipo de pensión, cuantía mensual, complementos recibidos, etc.). Esta muestra ha sido utilizada durante los últimos años para realizar diferentes tipos de estudios, como el análisis del mercado de trabajo (Cebrián et al., 2011), la contributividad del sistema de pensiones (Patxot et al., 2009) o su sostenibilidad (Moral-Arce et al., 2008).

La MCVL es una muestra muy amplia, al estar representadas un 4\% de las personas que guardan relación con la Seguridad Social cada año, ya sea como trabajadores activos o como pensionistas contributivos, por lo que las estimaciones realizadas son muy próximas a la realidad. No obstante, esta muestra no ha estado exenta de críticas en cuanto a su representatividad. El trabajo de Pérez-Salamero et al. (2015) concluye que la MCVL no es una réplica de la población objeto de estudio, para cierto tipo de investigaciones, especialmente cuando se seleccionan submuestras sobre determinado tipo de prestaciones.

Para comprobar la representatividad de la MCVL-2016, hemos comparado las principales magnitudes derivadas de la misma con los datos oficiales presentados en el Informe Estadístico del INSS (2016), tal y como se presenta en la Tabla 1. Como puede apreciarse, las diferencias entre ambas fuentes no son muy elevadas cuando se trata del número de pensiones de jubilación y viudedad, especialmente en el caso de las mujeres. En este tipo de pensiones, que generan más del $90 \%$ de los CDM, la sobrerrepresentación de la MCVL se sitúa por debajo del 1\%. Aun no siendo tan precisa la representación en las pensiones por incapacidad, pensamos que una sobrerrepresentación de alrededor del $3 \%$ no invalida las conclusiones de nuestro análisis. También puede observarse que las cuantías medias de todos los tipos de pensiones recibidas por las mujeres son muy representativas, con desviaciones que no superan el 0,5\%. En el caso de los hombres, las diferencias son siempre negativas y ligeramente mayores, pero por debajo del $0,7 \%$.

\section{Tabla 1. Diferencia entre la MCVL-2016 y el Informe Estadístico del INSS (\%)}

\begin{tabular}{|c|l|r|r|r|r|}
\hline \multicolumn{2}{|c|}{} & \multicolumn{1}{|c|}{ Jubilación } & \multicolumn{1}{c|}{ Incapacidad } & \multicolumn{1}{c|}{ Viudedad } & \multicolumn{1}{c|}{ Total } \\
\hline \multirow{3}{*}{ Número de Pensiones } & Mujeres & $0,6 \%$ & $3,1 \%$ & $0,7 \%$ & $0,8 \%$ \\
& Hombres & $1,5 \%$ & $3,0 \%$ & $2,6 \%$ & $1,7 \%$ \\
& Total & $1,2 \%$ & $3,1 \%$ & $0,8 \%$ & $1,2 \%$ \\
\hline \multirow{3}{*}{ Cuantía Media } & Mujeres & $0,5 \%$ & $-0,3 \%$ & $-0,2 \%$ & $0,0 \%$ \\
& Hombres & $-0,3 \%$ & $-0,7 \%$ & $-0,5 \%$ & $-0,4 \%$ \\
& Total & $0,0 \%$ & $-0,5 \%$ & $-0,3 \%$ & $-0,2 \%$ \\
\hline
\end{tabular}

FUENTE: Elaboración propia con datos de la MCVL-2016 y del Informe Estadístico del INSS 2016. 
La MCVL-2016 incluye, por primera vez, información muy útil para caracterizar y cuantificar los efectos del CDM. Al ser el primer año en que se ha comenzado a conceder este complemento, se han introducido dos nuevas variables en la muestra. Se trata del importe mensual total del CDM y del porcentaje del citado complemento (5\%, 10\% o 15\%). Gracias a esta información, podemos estimar de forma muy precisa, debido a la calidad de la muestra, las magnitudes más importantes de esta medida y su impacto diferencial en función de las características de las mujeres perceptoras.

Complementariamente, se ha realizado un contraste estadístico de independencia ( $\chi^{2}$-Pearson) entre diferentes parejas de variables, como el tipo de pensión que cobran las mujeres y la percepción o no del CDM y también el tipo de pensión con las distintas características sociodemográficas de las perceptoras del CDM (nivel educativo, número de hijos y régimen de cotización). En este tipo de contraste se pone a prueba la hipótesis nula de que las dos variables consideradas son independientes. Si el resultado del análisis reporta una significatividad límite ( $p$-valor) baja (menor al 1\%), ello señala que la hipótesis nula se ha de rechazar, lo que en este caso indica que las variables son dependientes. En los contrastes realizados siempre hemos rechazado la hipótesis nula, es decir que todas estas variables son dependientes, que es el resultado esperado en este tipo de prestaciones.

Hasta el momento los estudios con datos reales sobre el CDM no han sido posibles en España, aunque hay que destacar el trabajo pionero de Bonell y Devesa (2017) en el que se hace una primera estimación de la medida con los datos de la MCVL-2014, que todavía no contenía datos de las principales magnitudes del complemento.

El indicador brecha salarial de género utilizado en este trabajo, definido y estimado por el Instituto Nacional de Estadística (INE) (siguiendo a Eurostat y utilizando los datos de la Encuesta Anual de Estructura Salarial, 2015), es la diferencia entre el salario bruto por hora de los hombres y el de las mujeres, expresada como porcentaje del salario bruto por hora de los hombres. En cuanto a la brecha de género en las pensiones, utilizamos la definición de los estudios realizados por la Comisión Europea: diferencia entre la pensión media de los hombres y la de las mujeres, expresado como porcentaje de la pensión media de los hombres (Bettio et al. 2013). Para estimar esta brecha empleamos los microdatos de la MCVL-2016.

Por último, para analizar la variación en la desigualdad entre las pensiones de las mujeres debida al CDM, empleamos el indicador más utilizado como medida de desigualdad, el índice de Gini. Este índice se calcula (aplicando la definición del INE a las pensiones) a partir de las diferencias entre la distribución acumulada de la población pensionista (ordenada de menor a mayor según los importes de pensión que percibe) con los importes acumulados de pensión recibidos por los mismos; el resultado es un número entre 0 y 1 , en donde 0 se corresponde con la perfecta igualdad y una disminución del índice supondría una mejora en la distribución. Hubiera sido interesante el análisis gráfico mediante curvas de Lorenz, sin embargo, los cambios provocados por el complemento son tan reducidos que los resultados serían difícilmente visibles e interpretables. 


\section{Impacto socioeconómico de la medida}

Los datos contenidos en la MCVL-2016 nos permiten estimar el número total de mujeres que se ha podido beneficiar del CDM el primer año de aplicación, así como su distribución en función del tipo de pensión contributiva recibida y también comparar estos resultados con el resto de mujeres y hombres que han comenzado a percibir su pensión en 2016 y no han tenido derecho al nuevo complemento. En todo el trabajo no se han tenido en cuenta las pensiones contributivas por orfandad ni a favor de familiares, ya que estas pensiones no permiten obtener el CDM.

En la Tabla 2[A] observa que un total de 157.850 pensiones han recibido el CDM, lo que afecta al $58,4 \%$ del total de las altas de pensiones percibidas por mujeres en 2016. La mayor parte de los complementos se obtienen en las pensiones de viudedad (50,9\%), seguido de las pensiones de jubilación $(39,8 \%)$ e incapacidad $(9,2 \%)$. Por lo tanto, la pensión de viudedad es la pensión mayoritaria de las mujeres bonificadas, mientras que las mujeres sin complemento, son mayoritariamente las que han obtenido pensiones por derecho propio, ya sea por jubilación o incapacidad (50\% y 16,4\%, respectivamente).

En esta tabla también se aprecian las diferencias en las pensiones entre hombres y mujeres. Si bien el número total de altas es bastante similar en ambos géneros, la presencia de pensiones de viudedad es mucho mayor entre las mujeres (43,7\%) que entre los hombres $(6,3 \%)$. Esta diferencia es un importante factor explicativo de la brecha de género, ya que las pensiones de viudedad tienen una cuantía media muy inferior a las de jubilación o incapacidad.

La edad media de acceso a la pensión está muy próxima a los 65 años en el caso de jubilación, siendo muy inferior en las pensiones de incapacidad y superior en las de viudedad (Tabla 2[B]). Las mujeres que reciben el CDM acceden a la pensión a una edad superior a las que no reciben este complemento para todos los tipos de pensión. Si se compara el conjunto de mujeres con el de hombres, la edad media de acceso de las mujeres es bastante superior (66,9 años frente a 62) debido a que las mujeres se jubilan más tarde (sobre todo las que perciben CDM) y al mayor porcentaje de mujeres que perciben pensión de viudedad. 


\section{Tabla 2 Número de Pensiones y Edad, con y sin CDM. Altas 2016}

\begin{tabular}{|c|c|c|c|c|}
\hline \multicolumn{5}{|c|}{ [A] Número de pensiones } \\
\hline Categorías & Jubilación & Incapacidad & Viudedad & Total \\
\hline Mujeres con $\mathrm{CDM}^{*}$ & 62.900 & 14.575 & 80.375 & 157.850 \\
\hline Mujeres $\sin \mathrm{CDM}^{*}$ & 56.175 & 18.425 & 37.775 & 112.375 \\
\hline Total Mujeres & 119.075 & 33.000 & 118.150 & 270.225 \\
\hline Total Hombres & 187.925 & 52.450 & 16.250 & 256.625 \\
\hline Total (Hombres y Mujeres) & 307.000 & 85.450 & 134.400 & 526.850 \\
\hline & \multicolumn{4}{|c|}{ En el total de mujeres } \\
\hline Mujeres con CDM & $23,3 \%$ & $5,4 \%$ & $29,7 \%$ & $58,4 \%$ \\
\hline Mujeres sin CDM & $20,8 \%$ & $6,8 \%$ & $14,0 \%$ & $41,6 \%$ \\
\hline \multirow[t]{2}{*}{ Total Mujeres } & $44,1 \%$ & $12,2 \%$ & $43,7 \%$ & $100,0 \%$ \\
\hline & \multicolumn{3}{|c|}{ En el total de cada categoría } & \\
\hline Mujeres con CDM & $39,8 \%$ & $9,2 \%$ & $50,9 \%$ & $100 \%$ \\
\hline Mujeres sin CDM & $50,0 \%$ & $16,4 \%$ & $33,6 \%$ & $100 \%$ \\
\hline Total Mujeres & $44,1 \%$ & $12,2 \%$ & $43,7 \%$ & $100 \%$ \\
\hline Total Hombres & $73,2 \%$ & $20,4 \%$ & $6,3 \%$ & $100 \%$ \\
\hline Total (Hombres y Mujeres) & $58,3 \%$ & $16,2 \%$ & $25,5 \%$ & $100 \%$ \\
\hline \multicolumn{5}{|c|}{ [B] Edad media de acceso (años) } \\
\hline & Jubilación & Incapacidad & Viudedad & Total \\
\hline Mujeres con CDM & 65,1 & 53,6 & 74,1 & 68,6 \\
\hline Mujeres sin CDM & 64,1 & 49,9 & 72,4 & 64,6 \\
\hline Total Mujeres & 64,6 & 51,5 & 73,6 & 66,9 \\
\hline Total Hombres & 63,8 & 52,6 & 71,1 & 62,0 \\
\hline Total (Hombres y Mujeres) & 64,1 & 52,2 & 73,3 & 64,5 \\
\hline
\end{tabular}

${ }^{*} \chi_{2}^{2}=8.785 ; \alpha<1 \%$ valor crítico $=9,21$.

FUENTE: Elaboración propia con datos de la MCVL-2016.

A continuación, se analizan determinadas características sociodemográficas de las nuevas pensionistas en 2016. En primer lugar, en la Tabla 3, se distingue en función del número de hijos por mujer, determinante directo del porcentaje de complemento obtenido. Según las estimaciones realizadas, el complemento más común es el $5 \%$, que corresponde a madres de 2 hijos (en un $53,4 \%$ de los casos), seguido del 10\% (27,6\%), para madres de 3 hijos, y, finalmente, el $15 \%$ que aplican las madres de 4 hijos o más (19\% del total). Puede observarse que el complemento se presenta en las pensiones de jubilación y viudedad en una proporción muy similar para las madres con dos hijos 
(23,9\% y 22,9\%, respectivamente) y, lo que es más destacable, las mujeres con más de 2 hijos se concentran en mayor proporción en las pensiones de viudedad. Hay que apuntar que estas mujeres tienen una edad media en torno a los 74 años (Tabla 1[B]), pertenecientes a generaciones que han trabajado menos tiempo y han tenido más hijos. No podemos comparar el número de hijos con las mujeres que no han recibido el CDM, al no figurar este dato en la MCVL-2016.

\section{Tabla 3. Distribución de Pensiones percibidas por Mujeres con CDM según Número de hijos y Tipos de pensión. Altas 2016 (\% sobre el total)}

\begin{tabular}{|l|r|r|r|r|}
\hline Número de hijos [\% de CDM] & Jubilación & Incapacidad & Viudedad & \multicolumn{1}{|c|}{ Total } \\
\hline 2 hijos [5\%] & $23,9 \%$ & $6,6 \%$ & $22,9 \%$ & $53,4 \%$ \\
3 hijos [10\%] & $10,6 \%$ & $1,9 \%$ & $15,1 \%$ & $27,6 \%$ \\
4 hijos o más [15\%] & $5,3 \%$ & $0,7 \%$ & $13,0 \%$ & $19,0 \%$ \\
\hline Total 2 hijos o más & $39,8 \%$ & $9,2 \%$ & $50,9 \%$ & $100,0 \%$ \\
\hline
\end{tabular}

${ }^{*} \chi_{4}^{2}=6.672 ; \alpha<1 \%$ valor crítico $=13,28$.

FUENTE: Elaboración propia con datos de la MCVL-2016.

En cuanto a la distribución del CDM por niveles de estudio (Tabla 4), se observa que el complemento se concede en mayor medida a las mujeres sin estudios (52,1\% del total de quienes lo perciben), sobre todo a las pensionistas de viudedad (32,3\%), posiblemente porque son las que acceden con una edad media más elevada. Sin embargo, en jubilación predominan las perceptoras del complemento que han alcanzado el nivel primario u otros estudios superiores, que son las que tienen edades próximas a los 65 años. En relación al nivel de estudios de los otros pensionistas del sistema, las mujeres sin complemento tienen proporcionalmente mayores niveles de estudio que las mujeres con complemento, y los niveles de éstas son muy similares a los de los hombres.

\section{Tabla 4. Distribución de Pensiones según Niveles de estudios y} Tipos de pensión. Altas 2016 (\% sobre el total de la categoría)

\begin{tabular}{|l|r|r|r|r|r|r|}
\hline \multirow{2}{*}{ Nivel de estudios } & \multicolumn{4}{|c|}{ Mujeres con CDM } & Mujeres sin CDM & \multicolumn{1}{c|}{ Hombres } \\
\cline { 2 - 7 } & \multicolumn{1}{|c|}{ Jubilación } & Incapacidad & Viudedad & \multicolumn{1}{c|}{ Total } & \multicolumn{1}{c|}{ Total } & \multicolumn{1}{c|}{ Total } \\
\hline$<$ Primaria & $17,0 \%$ & $2,8 \%$ & $32,3 \%$ & $52,1 \%$ & $35,1 \%$ & $34,7 \%$ \\
Primaria & $14,0 \%$ & $4,2 \%$ & $12,4 \%$ & $30,6 \%$ & $32,7 \%$ & $34,7 \%$ \\
Secundaria & $6,3 \%$ & $1,5 \%$ & $3,5 \%$ & $11,2 \%$ & $20,3 \%$ & $20,4 \%$ \\
Superior & $2,4 \%$ & $0,6 \%$ & $1,4 \%$ & $4,4 \%$ & $9,4 \%$ & $7,5 \%$ \\
Sin Datos & $0,2 \%$ & $0,2 \%$ & $1,2 \%$ & $1,7 \%$ & $2,5 \%$ & $2,7 \%$ \\
\hline Total & $39,8 \%$ & $9,2 \%$ & $50,9 \%$ & $100,0 \%$ & $100,0 \%$ & $100,0 \%$ \\
\hline
\end{tabular}

${ }^{*} \chi_{6}^{2}=10.569 ; a<1 \%$ valor crítico $=16,81$.

FUENTE: Elaboración propia con datos de la MCVL-2016. 
La distribución del CDM por Regímenes de afiliación (Tabla 5), considerando los dos más importantes -Régimen General (RG) y Régimen Especial de Trabajadores Autónomos (RETA)- es significativamente más elevada en el RG $(79,8 \%)$, como lo es la participación de la población pensionista total en el mismo. Ahora bien, si consideramos la proporción de altas con complemento en cada régimen, perciben complemento el $62,5 \%$ de las altas de trabajadoras del RETA frente al $57,5 \%$ de las trabajadoras del RG. Esta diferencia favorable en el RETA procede de las altas por jubilación e incapacidad, lo que pone de manifiesto que las trabajadoras autónomas jubiladas en 2016 han tenido un número medio de hijos superior al de las asalariadas.

\section{Tabla 5. Distribución de Pensiones percibidas por Mujeres según Regímenes y Tipos de pensión. Altas 2016}

\begin{tabular}{|l|r|r|r|r|r|r|r|}
\hline \multirow{2}{*}{\multicolumn{1}{c|}{ Regímenes }} & \multicolumn{4}{|c|}{ Mujeres con CDM } & \multicolumn{2}{c|}{$\begin{array}{c}\text { Mujeres (\% sobre el total de cada } \\
\text { Régimen) }\end{array}$} \\
\cline { 2 - 8 } & Jubilación & $\begin{array}{c}\text { Incapaci- } \\
\text { dad }\end{array}$ & Viudedad & Total & con CDM & sin CDM & Total \\
\hline General & $31,2 \%$ & $7,7 \%$ & $40,8 \%$ & $79,8 \%$ & $57,5 \%$ & $42,5 \%$ & $100 \%$ \\
Autónomos & $8,6 \%$ & $1,5 \%$ & $10,1 \%$ & $20,2 \%$ & $62,5 \%$ & $37,5 \%$ & $100 \%$ \\
\hline Total & $39,8 \%$ & $9,2 \%$ & $50,9 \%$ & $100 \%$ & $58,4 \%$ & $41,6 \%$ & $100 \%$ \\
\hline
\end{tabular}

${ }^{*} \chi \_2{ }^{\wedge} 2=216 ; a<1 \%$ valor crítico $=9,21$.

FUENTE: Elaboración propia con datos de la MCVL-2016.

Por último, el gasto total de prestaciones correspondiente al complemento, según los datos de la MCVL-2016, habría ascendido a 64.303.802 € en el año 2016. Este importe es prácticamente el doble al estimado para el primer año de aplicación por Bonell y Devesa (2017), que era de 32.582.247 $€$ y también superior al estimado inicialmente por el MESS (2015), que ascendía a 51.616.278 €. El motivo de nuestras divergencias es que los otros trabajos no contaban todavía con datos de la MCVL-2016, viéndose obligados a realizar estimaciones menos precisas. El gasto del primer año de aplicación, que supone en torno al $0,05 \%$ del gasto total presupuestado en pensiones contributivas (118.942 millones de $€$ ), se irá incrementando a medida que se vayan acumulando las altas que se vayan produciendo en los años siguientes. Bonell y Devesa (2017) estiman que cuando el complemento se estabilice, 60 años después de su implantación, puede suponer más del $2 \%$ del gasto público en pensiones. 


\section{Diferencias de trato: posibles consecuencias discriminatorias}

El CDM se aplica exclusivamente a madres con al menos dos hijos. La medida excluye a determinadas mujeres con hijos y también a los padres. Diversos autores se plantean si esta diferencia de trato es discriminatoria (Gallego, 2016; De la Flor, 2016). En nuestra opinión, estas exclusiones serán socialmente discriminatorias si implican un trato de inferioridad a las personas excluidas, con consecuencias negativas relevantes. A continuación se analizan las personas excluidas por el complemento.

\subsection{Mujeres que ya eran pensionistas antes de 2016}

Este es un importante colectivo que ha quedado fuera de la aplicación del complemento. Su exclusión significa no reconocer su aportación demográfica que, en términos medios, corresponde a las generaciones de mujeres que han tenido más hijos que las siguientes, siendo las más afectadas por estructuras laborales y familiares más desfavorables y para las que la brecha de género en la pensión es superior. Se trata sobre todo de las madres del baby-boom (que lo fueron entre 1945-64) y las madres de la Generación X (de hijos nacidos entre 1965-79), cuyas tasas de fertilidad fueron, según datos del Banco Mundial (2017), de 2,88 y 2,78, más del doble que la actual tasa de fertilidad, que se sitúa en 1,34 en 2017. En este sentido, el CDM discrimina en función de la edad.

Si suponemos que la distribución de hijos por mujer de las pensiones causadas con anterioridad a 2016 es la misma que la de las altas con derecho a CDM, la proporción de las altas en 2016 que lo perciben $(58,4 \%$, tabla 2$)$ sería también la que corresponde al total de mujeres pensionistas de 2016 (3.842.500, estimadas con la MCVL-2016, eliminando las concurrencias de pensión). En tal caso, se estima que la aplicación actual del complemento excluye a 2.244 .020 mujeres por ser pensionistas antes de 2016.

\subsection{Madres de un solo hijo}

La posición del gobierno, al establecer el complemento para madres de al menos dos hijos, se justifica en la idea de que solo debe premiarse el esfuerzo de las madres que han tenido hijos por encima de la media. Sin embargo, no parece sensato plantear que las mujeres trabajadoras madres de un solo hijo no han contribuido a la demografía y al mantenimiento del sistema de pensiones, y su exclusión parece estar justificada principalmente en términos de coste financiero de la medida (Gallego, 2016). 
Sin duda esta exclusión es importante pero difícil de cuantificar, ya que la MCVL-2016 no proporciona este dato. No nos ha parecido una alternativa válida utilizar la Encuesta de Fecundidad de 1999 para estimar el número de pensionistas que han tenido un hijo, puesto que esta encuesta representa al conjunto de mujeres españolas y no a las que van a cobrar una pensión contributiva.

\subsection{Mujeres en familias monoparentales}

El CDM tampoco considera el sobresfuerzo que supone criar a los hijos en familias monoparentales, en las que se presentan mayores dificultades, tanto financieras como de reconocimiento social como madres solteras (CSEPT, 2015a: 6). Según la Encuesta Continua de Hogares de 2016, el 66,2\% de las madres que conviven solamente con hijos, lo hacen solo con un hijo. Por lo tanto, es muy posible que la mayor parte de las familias monoparentales no accedan al complemento.

\subsection{Mujeres sin derecho a pensión de jubilación}

El complemento excluye a las mujeres que han desarrollado solo trabajo doméstico o, en muchos casos, trabajo en la economía sumergida o en negocios familiares.

Según la Encuesta de Fecundidad de 1999, que entrevista a mujeres entre 15 y 49 años de edad en aquel año (que son las que en 2016 tienen entre 32 y 66 años), el 26\% de ellas se dedica a labores del hogar, siendo este grupo el que, en términos medios, más hijos tiene (Tabla 6). Estas mujeres van a verse discriminadas por partida doble. En primer lugar, porque tienen mayores dificultades para acceder a la pensión de jubilación y, en segundo lugar, porque no tienen derecho al CDM.

En cuanto a las mujeres ocupadas, las que más hijos tienen son las que trabajan por cuenta propia, seguidas de las asalariadas fijas, sin duda los dos colectivos que tendrán más posibilidades de recibir el CDM. Descartando las situaciones menos relevantes, destacan las asalariadas temporales y las que buscan empleo como las mujeres que menos hijos tienen en términos medios. A estos dos colectivos les será difícil alcanzar una pensión contributiva y, aunque lo logren, muchas de ellas quedarán excluidas del CDM al no haber tenido al menos dos hijos. 
Tabla 6. Número de hijos según actividad económica. Mujeres entre 15 y 49 años (1999)

\begin{tabular}{|l|c|c|}
\hline & Número de mujeres (\%) & Número de hijos (media) \\
\hline Labores del hogar & $26 \%$ & 1,97 \\
\hline Jubiladas, pensionistas & $1 \%$ & 1,52 \\
\hline Ocupadas por cuenta propia & $8 \%$ & 1,46 \\
\hline Ocupadas asalariadas fijas & $21 \%$ & 1,07 \\
\hline Otra situación & $2 \%$ & 0,98 \\
\hline Paradas buscando empleo & $11 \%$ & 0,84 \\
\hline Ocupadas otra situación & $2 \%$ & 0,83 \\
\hline Ocupadas asalariadas temporales & $14 \%$ & 0,67 \\
\hline Estudiantes & $17 \%$ & 0,04 \\
\hline Total \% (número) & $100 \%(10.165 .237)$ & 1,07 \\
\hline
\end{tabular}

FUENTE: Elaboración propia con datos de la encuesta de fecundidad (1999).

También hay mujeres que han trabajado fuera del hogar, pero que no alcanzan el periodo mínimo de 15 años para acceder a la pensión contributiva. El Informe sobre el Desarrollo del Pacto de Toledo del año 2008 (MTIN, 2008) estimaba que existían unas 120.000 personas, entre 60 y 65 años, que aún no tenían registrados 15 años de cotización real, de las que 30.000 eran hombres y 90.000 mujeres. A pesar de la importancia de este hecho diferencial de género, la Ley 40/2007 endureció el cómputo de la carencia pasando a exigir que los 15 años requeridos fueran de cotización real (sin el cómputo de las pagas extras como tiempo cotizado).

\subsection{Mujeres que acceden a pensiones no contributivas}

Se trata de cobertura asistencial, de cuantía inferior a la pensión contributiva, que perciben 301.511 mujeres por jubilación (a partir de los 65 años) o por incapacidad (MESS, 2017), al carecer de pensiones contributivas y de otros recursos. La proporción de mujeres que perciben estas pensiones es del $6 \%$ de la población de mujeres de 65 años o más.

Estas mujeres no tienen derecho al CDM, aunque pueden haber realizado la misma aportación demográfica que las pensionistas del sistema contributivo. Además, se trata de importes de pensión que están muy por debajo de la pensión mínima contributiva $(367,90 €$ y 636,10 € al mes en 2016, respectivamente), por lo que al no recibir el complemento la diferencia entre ambos tipos de pensión va a ser cada vez mayor. 


\subsection{Mujeres con jubilación anticipada voluntaria}

En la Seguridad Social, la edad establecida para percibir la pensión de jubilación contributiva se establece, con carácter general, entre los 65 y 67 años, según el período de cotización acumulado, de modo que, jubilarse antes de esa edad de referencia, supone anticipar la jubilación. Si la anticipación es voluntaria, no se tiene derecho al complemento, ni en el momento de causar alta ni cuando se alcanza la edad ordinaria de jubilación. El número de jubilaciones de este tipo en 2016 es importante: 12.500 mujeres (el 4,4\% de las altas), según los datos de la MCVL-2016.

También se excluye del complemento a jubilaciones anticipadas a la jubilación parcial, aunque es con carácter temporal hasta la jubilación plena. Según los datos de la MCVL-2016, ha afectado a 9.425 mujeres (el $3,3 \%$ de las altas).

Tampoco tienen derecho al complemento las funcionarias que se jubilan antes de la edad forzosa de jubilación, que es, en general, a los 65 años y, en particular, a los 70 para funcionarias de Cuerpos Docentes Universitarios, Magistradas, Juezas, Fiscales y Letradas de la Administración de Justicia que se jubilan antes de la edad forzosa, que son 70 años.

La exclusión es difícil de justificar, salvo por recortar el gasto y por la intención de penalizar o desincentivar el acceso anticipado (Gallego, 2016).

\subsection{Padres que han ejercido la paternidad de manera responsable}

El complemento presenta, en nuestra opinión, una discriminación de género, al excluir de la prestación a los padres, posiblemente incompatible con el Derecho Comunitario (Gallego, 2016; Ballester, 2016). En este sentido, el Tribunal de Justicia de la UE (TJUE) consideró no justificada la diferencia de trato derivada de la bonificación por hijo concedida en Francia a las madres funcionarias (Caso Griesmar), porque al concederse en la pensión no era una ayuda para afrontar las dificultades profesionales soportadas por las madres (De la Corte, 2016). Es obvio que el CDM español se opone al principio de igualdad de trato entre hombres y mujeres (Directiva 79/7/CEE), puesto que la ventaja no se vincula a las excepciones por educación de los hijos, no aporta soluciones a los problemas ni a las desventajas laborales y no es una medida transitoria.

Según Rodríguez (2016), cabría efectuar una "relectura" crítica de una medida de esta índole desde la perspectiva de los principios de igualdad y no discriminación por razón de sexo; de algún modo parece una renuncia al fomento de la corresponsabilidad y una asunción implícita del rol de la mujer como madre y responsable principal de las tareas de cuidado de los hijos y del hogar, que el CDM pretende a consolidar o perpetuar. Si aspiramos a que la corresponsabilidad en el cuidado de hijos, de personas enfermas o de mayores, no recaiga solo en las mujeres, hay que reconocer los 
mismos derechos cuando son los hombres quienes realizan esas tareas. La concesión de medidas discriminatorias (favorables en exclusiva a las madres) genera resultados contrarios al pretendido, al apostar por afianzar la idea de la preservación del reparto tradicional de roles entre hombres y mujeres. El complemento contradice la pretensión de corresponsabilidad.

La discriminación se produce en mayor medida para los padres varones que crían solos a sus hijos, por ejemplo, en caso de fallecimiento de la madre 0 en caso de parejas gais, porque no aplica el complemento ninguno de los padres.

Por consiguiente, si el complemento por maternidad se mantiene, sería conveniente generalizarlo para madres y padres, si no se quiere incurrir en problemas de discriminación y de contradicción con la defensa de una mayor corresponsabilidad familiar.

\subsection{Otras exclusiones}

El complemento tampoco contempla las situaciones de guarda con fines de adopción y el acogimiento familiar, pese a que disfrutan de la protección por maternidad (Rodríguez, 2016), ni a los cuidadores en familias reconstituidas. Por tanto, no se les reconoce el esfuerzo por cuidar de menores $\mathrm{u}$ otras personas dependientes.

Del mismo modo, no considera la mayor dedicación, el esfuerzo diferencial superior, que supone la discapacidad de hijos, ni la discapacidad de ascendientes o de otros familiares. No se tiene en consideración, dice Gallego (2016) "ninguna acción positiva para mitigar el coste adicional que supone la discapacidad para familias", ni hace alusión a la medida de la Ley 40/2003, de Protección a las familias numerosas, de computar como dos a cada hijo discapacitado.

Además, podría decirse que el complemento excluye a las mujeres sin hijos, en la medida en que éstas también sufren discriminación salarial, si el objetivo del mismo es también suavizar la brecha de género en pensiones (Bonell y Devesa, 2017). 


\section{Efecto del CDM sobre la brecha de género en pensiones}

Uno de los principales argumentos para defender el CDM es que esta medida es un instrumento útil para reducir la brecha de género de las pensiones, porque aproxima la pensión media de las mujeres a la de los hombres (Consejo de Ministros, 2015; Bonell y Devesa, 2017). A continuación, analizamos detalladamente las causas de la brecha y cuantificamos el efecto del complemento.

Según información publicada por el Parlamento Europeo (Davaki, 2016), las europeas ganan de media en 2012 alrededor de un 16,5\% menos que los hombres, pero la situación empeora cuando se jubilan, con pensiones un $40,2 \%$ inferiores. Este dato refleja que la situación de las mujeres mayores es peor que la conocida tesis general de que el sistema contributivo de pensiones reproduce las desigualdades de género que existen en el mercado laboral. La incidencia de la normativa de pensiones aumenta la diferencia de género (Vara, 2011; Errandonea, 2016).

En España, al igual que en el resto de países europeos, el punto de partida, a pesar de los importantes avances conseguidos en materia de igualdad laboral y de oportunidades, es la importante brecha salarial de género, consecuencia de la segregación horizontal y vertical en el mercado de trabajo (MESS, 2016). Según las estimaciones publicadas por el INE sobre datos en 2015, la brecha salarial, en salario hora es del 13,75\%. La diferencia salarial se agudiza en las generaciones de mayor edad (22,7\% en los trabajadores de 55 a 64 años y $48,6 \%$ para mayores de 65 años) y también es superior a la media en los contratos a tiempo parcial (14,5\%) frente a los de jornada a tiempo completo $(10,8 \%)$, debiéndose tener en cuenta que el trabajo a tiempo parcial está mucho más presente para las mujeres $(25,2 \%)$ que para los hombres $(7,9 \%)$.

La MCVL-2016 nos permite valorar la diferencia de género en las pensiones contributivas en España en 2016. En la Tabla 7 se presenta, en primer lugar, la brecha de género estimada para las pensiones $[A]$, diferenciando por tipos de pensión, y, en segundo lugar, las brechas correspondientes a los pensionistas -teniendo en cuenta que algunos de ellos perciben más de una pensión-, desglosando tanto por nivel educativo [B.1] como por tramos de edad [B.2].

En cuanto a la brecha en pensiones, estimada a partir de la pensión media por pensionista de la MCVL-2016, es del 37,7\%. Por tipos de pensión, la de jubilación muestra un resultado similar a la media $(36,6 \%)$, la de incapacidad presenta una distancia de género menos significativa $(17,2 \%)$ y la correspondiente a viudedad es negativa $(-37,2 \%)$, puesto que la brecha en este caso juega a favor de las mujeres ya que la base reguladora de la viudedad la generan, principalmente, cónyuges varones con salarios superiores. 
Si tenemos en cuenta la pensión media por pensionista, acumulando todas las pensiones percibidas por cada persona (por ejemplo, viudedad con jubilación o con incapacidad), la brecha da un resultado significativamente inferior $(29,3 \%)$. Esta reducción se debe al efecto de la concurrencia de las pensiones de viudedad con otros tipos de pensión, que perciben las mujeres de forma mayoritaria. Como cabe esperar, la brecha disminuye con el nivel educativo alcanzado, si bien continúa siendo un problema importante para las mujeres con estudios universitarios, que reciben unas pensiones un $19,4 \%$ inferiores a los hombres con ese mismo nivel de estudios. En cuanto a la división por tramos de edad se produce un efecto curioso. Si bien la brecha de género va aumentando con la edad, debido a que las diferencias de cotización se van reduciendo con el tiempo, esta brecha acaba siendo más baja en las edades más avanzadas. Esta reducción se explica por la concurrencia de las pensiones de viudedad con el resto de pensiones, que se produce especialmente en mujeres de edades avanzadas.

\section{Tabla 7. Brecha de género en las pensiones y en los pensionis- tas. MCVL-2016}

\begin{tabular}{|c|c|c|c|}
\hline Categorías & $\begin{array}{l}\text { Pensión Media } \\
\text { Mujeres (en €) }\end{array}$ & $\begin{array}{l}\text { Pensión Media } \\
\text { Hombres (en } € \text { ) }\end{array}$ & $\begin{array}{c}\text { Brecha } \\
\text { de Género }\end{array}$ \\
\hline \multicolumn{4}{|c|}{ [A] En pensiones. Por tipo de pensión } \\
\hline Jubilación & 766,39 & $1,208,72$ & $36,6 \%$ \\
\hline Incapacidad & 817,84 & 987,61 & $17,2 \%$ \\
\hline Viudedad & 649,86 & 473,81 & $-37,2 \%$ \\
\hline Total Pensiones & 715,29 & $1.147,53$ & $37,7 \%$ \\
\hline \multicolumn{4}{|c|}{ [B.1] En Pensionistas. Por nivel educativo } \\
\hline$<$ Primaria & 749,74 & $1.040,53$ & $27,9 \%$ \\
\hline Primaria & 874,16 & $1.234,68$ & $29,2 \%$ \\
\hline Secundaria & $1.223,23$ & $1.609,17$ & $24,0 \%$ \\
\hline Superior & $1.492,14$ & $1.852,06$ & $19,4 \%$ \\
\hline Sin Datos & 682,19 & 950,30 & $28,2 \%$ \\
\hline Total Pensionistas & 849,89 & $1.201,70$ & $29,3 \%$ \\
\hline \multicolumn{4}{|c|}{ [B.2] En Pensionistas. Por tramos de edad } \\
\hline$<50$ años & 755,85 & 856,61 & $11,8 \%$ \\
\hline 50-64 años & 930,61 & $1.235,79$ & $24,7 \%$ \\
\hline $65-80$ años & 902,62 & $1.297,73$ & $30,4 \%$ \\
\hline$>80$ años & 762,93 & $1.020,88$ & $25,3 \%$ \\
\hline Total Pensionistas & 849,89 & $1.201,70$ & $29,3 \%$ \\
\hline
\end{tabular}

FUENTE: Elaboración propia con datos de la MCVL-2016. 
Otra cuestión interesante es la distribución de la brecha de género en la pensión media por pensionista entre todos los pensionistas en 2016. Los hemos ordenado de menor a mayor según sus importes de pensión y dividido en decilas, diferenciado dos series, hombres y mujeres, para poder calcular la brecha de género por pensionista existente en cada una de estas decilas. En la Figura 1 puede apreciarse, como idea principal, que esta brecha crece conforme va aumentando la pensión media, llegando a ser igual o superior al $36,9 \%$ para un $40 \%$ de las pensionistas, las que están situadas en niveles de pensión medios-altos (entre la decila 6 y 9). No obstante, llama la atención los dos casos que se desmarcan de esa tendencia. Observamos, por un lado, la alta brecha en la decila $1(30,8 \%)$, que pone de manifiesto lo reducidas que son, en términos relativos, las pensiones de las mujeres que menos pensión reciben, en relación a la decilas las decilas 2 y 3 (9,4\% y 11,8\%, respectivamente), cuyos importes medios son prácticamente coincidentes con los importes de pensiones mínimas (pensiones con complemento de mínimos), sobre todo para las mujeres. Por otro lado, en el extremo superior de la distribución, la brecha de la decila $10(23,4 \%)$ es inferior a la brecha del total $(29,3 \%)$, por el efecto igualador del límite a la pensión máxima. Es de destacar, pues, la importancia que tienen los límites mínimos y máximos de pensión en la reducción de la brecha de género en pensiones.

\section{Figura 1. Brecha de género y pensiones medias por pensionis- ta, por decilas. MCVL-2016}

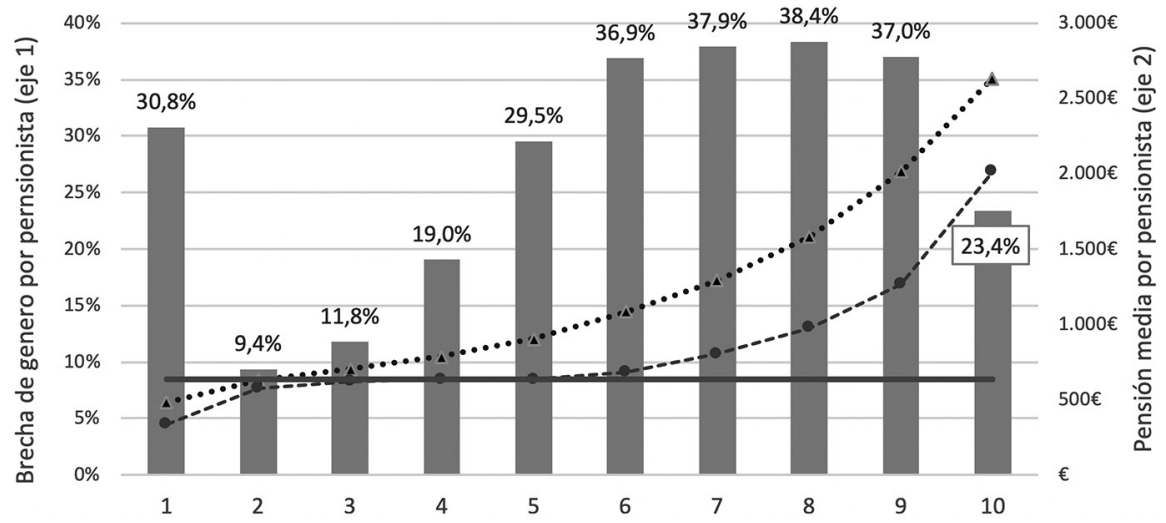

Brecha (eje 1) ••^••Pensión Hombres (eje 2) - -•- Pensión Mujeres (eje 2) — Pensión mínima (eje 2

FUENTE: Elaboración propia con datos de la MCVL-2016.

Una vez analizada la situación de la brecha en pensiones, nos preguntamos por el efecto que ha tenido el CDM sobre dicha brecha. En la primera parte de la Tabla 8 se observa que ha originado un pequeño efecto positivo en la reducción de la brecha total, 0,1 puntos porcentuales (p.p.), comparando importes de pensión media del total de pensiones, y 0,2 p.p., si consideramos la pensión media por pensionista. 
Si tenemos en cuenta solo las altas de pensión en 2016 (Tabla 8[B]), los resultados mejoran sustancialmente: la brecha con CDM entre los nuevos pensionistas es el $28,9 \%$ frente al $37,7 \%$ del total. Esta reducción se debe, sobre todo, a que mejoran las pensiones de las nuevas pensionistas (-6,4 p.p. comparando las brechas sin complemento de todas las pensiones y de las altas) y al efecto del propio complemento, que solo cobrarán las nuevas pensionistas (-2,5 p.p.). Conviene destacar que en viudedad el efecto favorable es mayor, probablemente porque las nuevas pensiones son más altas.

Los resultados de las altas pueden ser un indicador de cuál hubiera sido el efecto del CDM si se hubiese concedido a todas las pensiones o pueden interpretarse como marcadores de la tendencia del sistema, siempre que las pautas de comportamiento del complemento se mantengan, pero de momento no actúan de manera decisiva para cerrar la brecha de género, como se ha afirmado por fuentes oficiales (CSEPT, 2015a).

\section{Tabla 8. Efecto del CDM sobre la brecha de género en las pen- siones y en la pensión por pensionista. MCVL-2016}

\begin{tabular}{|c|c|c|c|c|c|}
\hline & \multicolumn{5}{|c|}{ [A] Todas las pensiones 2016} \\
\hline & \multicolumn{4}{|c|}{ Total (pensión media) } & \multirow{2}{*}{$\begin{array}{c}\text { Total (pensión media } \\
\text { por pensionista) }\end{array}$} \\
\hline & Jubilación & Incapacidad & Viudedad & Total & \\
\hline Brecha sin CDM & $36,7 \%$ & $17,4 \%$ & $-36,8 \%$ & $37,8 \%$ & $29,5 \%$ \\
\hline Brecha con CDM & $36,6 \%$ & $17,2 \%$ & $-37,2 \%$ & $37,7 \%$ & $29,3 \%$ \\
\hline \multirow[t]{4}{*}{ Diferencia Brecha } & $-0,1 \%$ & $-0,2 \%$ & $0,4 \%$ & $-0,1 \%$ & $-0,2 \%$ \\
\hline & \multicolumn{5}{|c|}{ [B] Altas 2016} \\
\hline & \multicolumn{4}{|c|}{ Total (pensión media) } & Total (pensión media \\
\hline & Jubilación & Incapacidad & Viudedad & Total & por pensionist \\
\hline Brecha sin CDM & $23,1 \%$ & $19,5 \%$ & $-50,2 \%$ & $31,4 \%$ & $28,7 \%$ \\
\hline Brecha con CDM & $20,9 \%$ & $17,6 \%$ & $-58,4 \%$ & $28,9 \%$ & $26,5 \%$ \\
\hline Diferencia Brecha & $-2,2 \%$ & $-1,9 \%$ & $8,2 \%$ & $-2,5 \%$ & $-2,2 \%$ \\
\hline
\end{tabular}

FUENTE: Elaboración propia con datos de la MCVL-2016.

En el caso español, al igual que en el resto de Europa, la brecha en pensiones es superior a la brecha salarial. La diferencia puede verse si tomamos como datos representativos de esta situación la brecha salarial de 55 a 64 años, que es del $21 \%$, y la brecha en pensiones, que, para ese mismo intervalo de edad, se sitúa en torno al 27\% (MCVL-2016). Por tanto, la brecha de género en las pensiones de jubilación empeora en relación a la de los salarios. ¿Cuáles son los principales condicionantes de estas diferencias? 


\subsection{Incidencia de las bases de cotización en la brecha de género en las pensiones}

Como estiman Muñoz de Bustillo y Pinto (2018), a partir de la MCVL-2015, las mujeres tienen una base media de cotización inferior a la de los hombres, para todas las cohortes objeto de estudio (nacimientos entre 1946 y 1995), aumentando la diferencia a medida que transcurre la vida laboral, cuando los salarios son más altos, correspondiendo el máximo a los tramos de edad entre los 50 y 60 años. El denominado techo de cristal explica, según los citados autores, las mayores diferencias salariales en los tramos altos de la distribución salarial.

Un factor que explica estas diferencias en las bases de cotización es que la mayoría de los contratos a tiempo parcial recaen en trabajadoras y que el trabajo a tiempo parcial está menos remunerado que el trabajo a tiempo completo. Además, las mujeres se ven afectadas en mayor medida por la aparición de lagunas en su cotización superiores a dos años. Por lo tanto, su base reguladora se ve reducida con carácter adicional a las diferencias salariales. En conjunto, la diferencia en base reguladora media a tiempo completo es un 7\% inferior para las mujeres (MESS, 2016).

\subsection{Incidencia del periodo de cotización en la brecha de género en pensiones}

Las carreras de cotización de las mujeres son en general más cortas que las de los hombres. Según los datos del MESS (2015), en 2014 las mujeres cotizaron una media de 31,98 años frente a 40,11 en el caso de los hombres en el Régimen General. La diferencia es todavía mayor en el caso de los Régimen de Autónomos (26,86 frente a 38,52). Hay que considerar que la penalización actual por año no cotizado alcanza 2,52 puntos porcentuales, lo que supone generar porcentajes de pensión entre el $20,3 \%$ y el 30,3\% inferiores a los de los hombres, para el Régimen General y Autónomos, respectivamente.

La duración de la vida laboral es significativamente inferior para las mujeres, a pesar de la inclusión en sus carreras de períodos considerados por la ley como cotizados en las altas de jubilación, aunque la distancia tiende a acortarse en el tiempo para las sucesivas generaciones. Según muestran Cebrián y Moreno (2015), la diferencia en los períodos de cotización es menor para las edades inferiores y crece con la edad, hasta llegar a alcanzar una diferencia superior a los 5 años en torno a las edades más avanzadas. A pesar de que las mujeres incluidas en su estudio han tenido una mayor continuidad laboral en las edades más próximas a la jubilación, han cotizado menos tiempo que los hombres de su generación, lo que repercutirá negativamente en el momento de acceso a sus prestaciones por jubilación.

Un factor explicativo de estas diferencias es la incidencia en el empleo de la existencia de hijos: en general, la tasa de empleo es función creciente del número de hijos para los hombres y decreciente para las mujeres. Como se observa en la Tabla 9, las tasas de empleo en 2015 de las mujeres (entre 
25 y 49 años), con hijos menores de 12 años, son menores a las tasas de empleo de las mujeres de la misma edad sin hijos (-6,2 p.p.). En el caso de los hombres sucede lo contrario, sus tasas de empleo son superiores si tienen hijos (5,3 p.p.). Este comportamiento se repite a medida que aumenta el número de hijos, de una manera escalonada, hasta los 2 hijos. A partir del tercer hijo, el empleo cae significativamente tanto para las mujeres como para los hombres.

\section{Tabla 9. Diferencia en la tasa de empleo para la población entre 25 y 49 años, por sexo y por número de hijos (en puntos porcen- tuales). 2015}

\begin{tabular}{|l|r|r|}
\hline & Hombres & Mujeres \\
\hline Diferencia con hijos y sin hijos & 5,3 & $-6,2$ \\
\hline Diferencia 1 hijo y sin hijos & 0,8 & $-2,7$ \\
\hline Diferencia 2 hijos y 1 hijo & 0,8 & $-2,7$ \\
\hline Diferencia 3 o más hijos y 2 hijos & $-9,8$ & $-18,4$ \\
\hline
\end{tabular}

FUENTE: Elaboración propia con datos de INE-Empleo (actualizado 17/11/2016).

\section{Efecto del CDM sobre la desigualdad en las pensiones de las mujeres}

Además del efecto sobre la brecha de género en las pensiones, el CDM también va a tener un efecto sobre la situación económica de las distintas mujeres. Este efecto tiene su importancia porque no todas las mujeres van a recibir el complemento y también porque las perceptoras van a beneficiarse de una cuantía distinta, en función de su número de hijos y de su pensión previa.

La aplicación de cualquiera de los porcentajes previstos sobre importes crecientes de pensión previa, aumenta nominalmente más la pensión en las pensiones más altas. Además, las pensiones mínimas solo aplican el CDM sobre la pensión previa al complemento de mínimos, mientras que las pensiones más altas pueden recibir el complemento y situarse por encima de la pensión máxima. Para cuantificar este efecto, partimos de los datos de la MCVL-2016, y estimamos cómo se distribuye el CDM entre las mujeres que reciben este complemento en 2016, dividiéndolas por decilas de pensión.

Como se aprecia en la Figura 2, las pensiones obtienen complementos de importe superior a medida que la pensión crece, salvo algún caso puntual. El complemento se calcula sobre el importe de la pensión y, aunque los porcentajes se aplican proporcionalmente, resultan más beneficiadas en valo- 
res absolutos las mujeres con pensiones altas, incluso puede darse el caso de que se beneficien más las mujeres con salarios elevados que las que han tenido más hijos. Según explica Rodríguez (2016), aunque el porcentaje es superior cuantos más hijos ha tenido la mujer, el hecho de tomar como base la cuantía de la pensión da lugar a que resulten más beneficiadas las mujeres con salarios elevados, que es probable que hayan sufrido menos dificultades para compatibilizar la vida laboral y familiar.

El sistema de los porcentajes ha sido ampliamente criticado. No compensa las desventajas profesionales de las madres, sino que beneficia a quienes han duplicado sus esfuerzos compatibilizando trabajo retribuido y doméstico (De la Flor, 2016) y, sobre todo, a quienes han podido hacerlo compatible porque su buena y estable situación laboral les ha permitido contratar servicio doméstico y permanecer en sus puestos de trabajo. En términos nominales, el CDM beneficia claramente a las mujeres mejor situadas en el mercado laboral, manteniendo su buena situación también como pensionistas.

\section{Figura 2 Importe medio mensual del CDM por decilas de pen- sión (en euros). Altas en 2016}

119,96

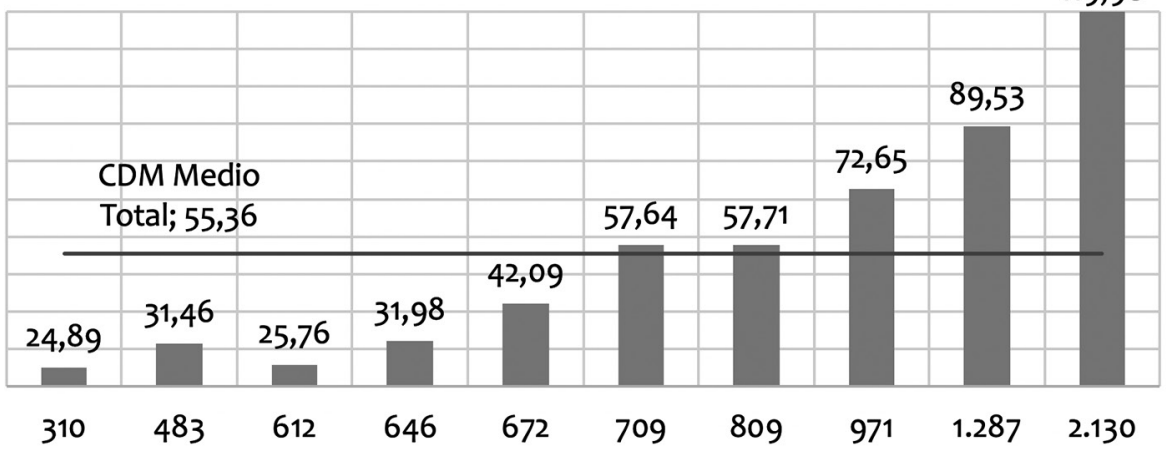

Pensión Media por decilas de pensión

FUENTE: Elaboración propia con datos de la MCVL-2016.

Otra cuestión es el efecto que tiene el complemento sobre la desigualdad entre las mujeres. Si todas las mujeres recibieran el mismo porcentaje de complemento, el efecto sobre la desigualdad sería nulo, ya que su pensión se vería incrementada en la misma proporción en todos los casos. Sin embargo, existen mujeres que no se benefician del CDM o que reciben diferente porcentaje en función del número de hijos.

Para estimar el efecto sobre la desigualdad, hemos calculado los Índices de Gini de las pensionistas, antes y después de recibir el complemento. En la Tabla 10 se presentan los resultados totales 
de las mujeres pensionistas y también los correspondientes a los tres grupos de pensiones: jubilación, incapacidad y viudedad.

El principal resultado que se observa en todos los casos analizados es que los cambios en la distribución son muy poco significativos y, por tanto, que los efectos redistributivos del complemento son muy reducidos. Los resultados concretos van a depender del colectivo de mujeres analizado y del tipo de pensión percibida.

[A] Si se analiza únicamente el grupo de mujeres que han recibido el complemento, el efecto redistributivo es tan pequeño $(-0,35 \%)$ que puede afirmarse que el CDM es prácticamente neutral. No obstante, se produce una pequeña reducción de la desigualdad, lo que indicaría que las mujeres con más hijos (las que reciben un porcentaje de CDM mayor) son las que tienen derecho a pensiones algo más bajas.

[B] Si se tienen en cuenta todas las altas en pensiones de 2016, también se produce un pequeño efecto de reducción de la desigualdad $(-0,85 \%)$. En este caso, se pone de manifiesto que las mujeres con derecho al complemento perciben pensiones algo más bajas que las mujeres sin complemento.

[C] No obstante, el efecto global del complemento para el total de mujeres pensionistas es de un pequeño empeoramiento en la distribución (0,14\%). Este cambio de signo se debe a que el complemento se ha aplicado solo sobre las altas, que corresponden a pensiones medias superiores a las que empezaron a recibirse en años anteriores.

$[A, B, C]$ Por tipos de pensión, el complemento aumenta la desigualdad en viudedad, posiblemente porque se aplica en menor proporción sobre las pensiones inferiores y no se aplica en caso de concurrencia con jubilación. En la pensión de jubilación el cambio en la distribución sigue la pauta general comentada anteriormente: es levemente progresivo entre las altas y ligeramente regresivo para el conjunto de pensionistas. Por último, la pensión de incapacidad muestra una tenue mejora en la distribución en todos los casos. 


\section{Tabla 10. Efectos distributivos del complemento entre las mujeres. Índice de Gini (\%)}

\begin{tabular}{|c|c|c|c|c|}
\hline \multirow[b]{3}{*}{ Gini $\sin C D M$} & \multicolumn{4}{|c|}{ (A) Altas 2016-Mujeres con derecho al CDM } \\
\hline & Jubilación & Incapacidad & Viudedad & Total Pensionistas \\
\hline & $31,63 \%$ & $31,03 \%$ & $23,16 \%$ & $28,55 \%$ \\
\hline Gini con CDM & $31,59 \%$ & $30,87 \%$ & $23,38 \%$ & $28,45 \%$ \\
\hline Diferencia & $-0,04 \%$ & $-0,16 \%$ & $0,22 \%$ & $-0,10 \%$ \\
\hline Variación & $-0,12 \%$ & $-0,52 \%$ & $0,97 \%$ & $-0,35 \%$ \\
\hline \multicolumn{5}{|c|}{ (B) Altas 2016} \\
\hline \multirow[b]{2}{*}{ Gini sin CDM } & Jubilación & Incapacidad & Viudedad & Total Pensionistas \\
\hline & $33,03 \%$ & $32,55 \%$ & $24,26 \%$ & $33,50 \%$ \\
\hline Gini con CDM & $32,60 \%$ & $32,35 \%$ & $24,56 \%$ & $33,22 \%$ \\
\hline Diferencia & $-0,43 \%$ & $-0,20 \%$ & $0,30 \%$ & $-0,28 \%$ \\
\hline \multirow[t]{3}{*}{ Variación } & $-1,32 \%$ & $-0,62 \%$ & $1,24 \%$ & $-0,85 \%$ \\
\hline & \multicolumn{4}{|c|}{ (C) Todas las pensiones 2016} \\
\hline & Jubilación & Incapacidad & Viudedad & Total Pensionistas \\
\hline Gini $\sin$ CDM & $32,97 \%$ & $31,83 \%$ & $24,28 \%$ & $28,05 \%$ \\
\hline Gini con CDM & $33,02 \%$ & $31,82 \%$ & $24,33 \%$ & $28,09 \%$ \\
\hline Diferencia & $0,05 \%$ & $-0,01 \%$ & $0,05 \%$ & $0,04 \%$ \\
\hline Variación & $0,14 \%$ & $-0,04 \%$ & $0,23 \%$ & $0,14 \%$ \\
\hline
\end{tabular}

FUENTE: Elaboración propia con datos de la MCVL-2016.

En conjunto no puede afirmase, como se ha hecho en alguna ocasión, que el complemento aumenta las diferencias entre las pensionistas del sistema contributivo, ni que agranda la brecha en pensiones entre ellas, quizás bajo un planteamiento erróneo de la pretensión de mecanismos progresivos de distribución. Con los resultados obtenidos, podemos afirmar que su efecto redistributivo en el sistema contributivo es muy poco significativo y su signo dependerá del tipo de pensión analizada y del colectivo de mujeres que se tenga en cuenta en la comparación. 


\section{CDM y Políticas de igualdad y pensiones}

De acuerdo con la legislación comunitaria y española referida en la introducción, las políticas públicas deben respetar y promover la aplicación del principio de igualdad de género y, aunque se permiten determinadas excepciones (acciones positivas a favor de las mujeres), éstas deben justificarse y ser de carácter transitorio.

En este sentido, la corriente principal de pensamiento (mainstreaming) defiende la orientación de políticas género desde las acciones positivas (políticas compensatorias) a las actuaciones para eliminar las causas de las desigualdades. Como dice Pazos (2013), es difícil justificar las denominadas políticas positivas, porque generan desigualdad de trato a las personas y porque no son efectivas.

Se sostiene que si el sistema de pensiones compensa las diferencias de género en el mercado laboral no se logrará más que reforzar los papeles tradicionales del hombre y la mujer; la concesión de mayores derechos de pensión por criar a los hijos a las mujeres que a los hombres puede ser un incentivo económico para mantener los papeles tradicionales del hombre y de la mujer, y desanimar a que las mujeres se mantengan fuera del mercado laboral formal y a los hombres a interrumpir sus carreras profesionales (Consejo de la UE, 2003; Pazos, 2013; Tinios et al., 2015). De hecho, estas medidas están siendo interpretadas de manera restrictiva por el TJUE, en el sentido que debe aplicarse la igualdad de trato entre madres y padres, y la revisión de la normativa comunitaria también está modificándose en el mismo sentido (Directiva 96/97/CE).

Desde esta visión se priorizan el establecimiento de auténticas políticas de igualdad, que creen oportunidades para que mujeres y hombres desarrollando su pleno potencial: 1) políticas en el mercado laboral que eliminen las diferencias de género, relativas al acceso al empleo, salarios y promoción en el puesto de trabajo, mejorando la flexibilidad horaria y la racionalidad de la jornada de trabajo y 2) políticas de conciliación de vida familiar y laboral, destinadas a todos los trabajadores, que integren a los padres y madres en la corresponsabilidad del cuidado de los hijos (servicios públicos de ayuda y de educación infantil de calidad, permisos parentales no transferibles de igual duración para padres y madres y medidas de sensibilización en la responsabilidad familiar compartida).

No obstante, en el corto y medio plazo, de alguna manera hay que actuar hasta que dichas políticas tengan efecto sobre las diferencias actuales. En el sistema de pensiones español, como ya hemos visto, la brecha de género de las pensiones contributivas es superior a la que corresponde a las diferencias salariales, porque el proceso de conversión de salario en derecho a pensión agranda la desigualdad (discriminación indirecta). En este marco es importante tener en cuenta: 
$1^{\circ}$ La obligación de realizar una valoración del impacto de género de las nuevas disposiciones normativas (Ley 30/2003) y la necesidad de estudiar la introducción de compensaciones en caso de que se éstas aumenten la desigualdad.

Ejemplos recientes del incumplimiento de esta obligación son las reformas de ampliación del período del cálculo de la base reguladora (de 15 a 25 años) y de encarecimiento de la obtención del porcentaje de pensión (Ley 27/2011), que perjudican sobre todo a las carreras incompletas, por tanto, tienen mayores efectos sobre las mujeres y son contrarias a la reducción de la brecha de género (Salvador, 2018). Estas medidas se están implantado sin haber analizado sus consecuencias.

$2^{\circ}$ La importancia de valorar y revisar los potentes instrumentos de igualación del sistema de pensiones, las rentas mínimas, pudiéndose considerar como tales: las pensiones no contributivas, los complementos a mínimos de pensiones contributivas y las pensiones de viudedad. Se trata de prestaciones que actualmente perciben mayoritariamente las mujeres y que reducen la brecha de género en pensiones, aunque deberían modificarse sustancialmente, reunificando prestaciones y revisando sus importes y las restrictivas condiciones de concesión.

$3^{\circ}$ El establecimiento de acciones positivas de carácter temporal, concedidas de forma indistinta a los hombres y a las mujeres y que no fomenten la permanencia ininterrumpida de la mujer en el hogar.

Actualmente el sistema de pensiones español contiene una serie de medidas específicas dirigidas a paliar los posibles efectos negativos de las interrupciones de la cotización, relacionados con la tenencia y cuidado de los hijos, y que benefician particularmente a las mujeres. Se trata de compensar su menor tasa de ocupación y sus menores carreras de cotización: relleno de lagunas por parto, reconocimiento de periodo de cotización por interrupciones en la cotización y por excedencias voluntarias y el cómputo mejorado del periodo de cotización en el trabajo a tiempo parcial. El CDM se enmarca también entre estas medidas.

El complemento ha sido ampliamente criticado, tanto por los representantes políticos como por los expertos (CSEPT, 2015a; 2015b; 2017), y también por diversos estudios (De la Flor, 2016; Gallego, 2016; Rodríguez, 2016). En principio, la justificación de su implantación no responde de manera directa a ningún mandato de las instancias e instituciones a las que apelan sus artífices, ni a políticas similares implantadas en otros países de la UE. No se ha realizado siguiendo la Recomendación $17^{\mathrm{a}}$ del Informe del Pacto de Toledo 2011 (MTIN, 2011) ni las recomendaciones de la UE en el Pacto Europeo por la Igualdad de Género (2011-2020) (Consejo de la UE, 2011), en las que se apela, en primera instancia, a la implementación de políticas para reducir las diferencias de género en el empleo y en los salarios, a la mejora de la conciliación y, específicamente en el sistema de pensiones, al reconocimiento de carreras de cotización por los períodos de atención a los hijos y a otros dependientes. 
En este sentido, la medida española no mejora las carreras de cotización, por lo que no se ha diseñado como los complementos a pensiones de otros países, que, en líneas generales, afectan a la pensión básica, son de importe fijo o solo mejoran el período de cotización. Según la información facilitada por el propio Informe sobre el complemento por maternidad (MESS, 2015: 13), en Francia la mejora se establece solo para quienes han tenido al menos 3 hijos: en la pensión base, al fijarse la tasa completa del $50 \%$, y, en la pensión complementaria (de jubilación o viudedad), con un complemento del 10\% (Frericks et al., 2007). En Luxemburgo, la prestación denominada fofait d'éducation, de $86,54 €$ mensuales por cada hijo, se concede a la persona que se haya encargado principalmente de la educación de los hijos; es incompatible con el complemento de mínimos y con los baby-years por un mismo hijo (los baby-years son años de cotización reconocidos en caso de interrupción de la actividad laboral, con máximo de 2 años o de 4 años en casos especiales). Alemania reconoce 3 años de cotización por hijo para el reconocimiento de la pensión básica (Frericks et al., 2007; Consejo de la UE, 2003 y 2015; Bonell y Devesa, 2017).

Se ha pretendido justificar la implementación del CDM por su capacidad para compensar los perjuicios invisibles a las carreras de cotización derivados del cuidado de los hijos y por el reconocimiento social a la maternidad. Sin embargo, esos perjuicios suelen consistir en abandonos forzados por incompatibilidades horarias o por la ralentización de las carreras profesionales, consecuencia de una defectuosa regulación en España de los mecanismos de conciliación y corresponsabilidad (Ballester, 2016). Por otro lado, la experiencia internacional demuestra que la solución a los problemas demográficos está en proporcionar a las mujeres la libertad y el entorno adecuado para que -en todos los países, en todos los niveles educativos y en todos los niveles de renta- puedan tener los hijos que deseen (Pazos, 2011).

La medida adoptada en España no aborda las causas del problema de las diferencias de género, ni es el suplemento de otras medidas más directas que abordan la base del problema. Como medida aislada, en nuestra opinión, es insuficiente para resolver la fuerte discriminación en el empleo y en las pensiones y puede ser contraproducente. El reconocimiento de la contribución demográfica no reduce la importante necesidad de ayudas inmediatas (Rodríguez, 2016) para que pueda desarrollarse la maternidad y paternidad libremente y de manera compatible con el trabajo, es ineficaz e ineficiente como estímulo de comportamientos que se verán compensados a muy largo plazo (Gallego, 2016) y, el sistema de premios exclusivo para las mujeres, puede verse como una medida anacrónica, como incentivo al mantenimiento del reparto tradicional de roles (De la Flor, 2016) contrario al principio de igualdad.

La mayoría de estas acciones son, en nuestra opinión, cuestionables e insuficientes. Pueden tener a corto plazo un efecto positivo de reducción de la brecha de género en las pensiones contributivas, pero pueden hacer que el problema original persista y sea más difícil de cambiar (Tinios et al., 2015 y Pazos, 2013). Disminuir las diferencias de género requiere, con carácter prioritario, mejorar la situación de las mujeres olvidadas, las más perjudicadas, que son, las mujeres sin pensión y las que perciben pensiones no contributivas (CSEPT, 2015b). 


\section{Conclusiones}

El CDM es una medida puesta en práctica en 2016 con el objetivo de reducir la brecha de género en las pensiones y también de reconocer socialmente la contribución de la maternidad al sistema de pensiones.

Gracias a los datos de la MCVL-2016 hemos podido cuantificar esta medida, que ha afectado al $58,4 \%$ de las nuevas pensionistas. Las mujeres que más se han beneficiado de esta medida son las que cobran pensiones de viudedad $(50,9 \%)$, seguidas por las pensiones de jubilación $(39,8 \%)$ e incapacidad $(9,2 \%)$. En cuanto a las características sociodemográficas de las perceptoras, el $53,4 \%$ de los complementos corresponde a madres con 2 hijos, mientras que las madres con más de 2 hijos se concentran en mayor medida en las pensiones de viudedad. Las mujeres sin complemento son mujeres con niveles de estudio superiores a las que perciben complemento, destacando el caso de viudas sin estudios, que suponen un 32,3\% del total de mujeres con CDM. También se comprueba que las trabajadoras autónomas han tenido más hijos y, por lo tanto, reciben el CDM en mayor proporción $(62,5 \%)$ que las trabajadoras por cuenta ajena $(57,5 \%)$. En cuanto al gasto estimado de esta medida, ha ascendido a 64 millones de euros en 2016 (aproximadamente el 0,05\% del gasto en pensiones contributivas), cifra que irá aumentando a medida que el complemento se vaya extendiendo a futuras pensionistas.

Entre las principales críticas a esta medida destacamos, en primer lugar, las diferencias de trato que genera. No se explica cómo pretende ser un reconocimiento a la maternidad si discrimina a muchas madres, excluyendo a las mujeres que ya eran pensionistas antes de 2016, que son las más afectadas por estructuras familiares y laborales más desfavorables. Además, también deja fuera a las madres de un solo hijo, a las jubiladas anticipadamente por el sistema voluntario, a las del sistema no contributivo y a las que no acceden a ninguna pensión y es contraria al principio de igualdad entre hombres y mujeres, en cuanto a que discrimina a los padres, pudiendo generar efectos contraproducentes sobre la igualdad real en el mercado de trabajo y en el ámbito familiar.

Por otro lado, una de las críticas más severas es que, al aplicarse como un porcentaje de la pensión previa, asigna un incremento a la pensión de importe creciente a medida que la pensión aumenta y no se limita su importe cuando se alcanza el tope máximo de pensión. De este modo, el sistema, frente a las opciones utilizadas en otros países, beneficia nominalmente más a las mujeres con pensiones altas. 
Por último, sus efectos sobre la reducción de la desigualdad son poco significativos. Como medida de igualdad entre hombres y mujeres, puede decirse que el CDM ha reducido la brecha de género de las altas en 2016 (2,2 puntos porcentuales), pero que su efecto en el conjunto del sistema es muy pobre (0,2 p.p.). En este sentido, queda mucho trabajo por hacer, ya que la brecha en pensiones continúa siendo muy elevada en España (29,3\%).

Los efectos entre las pensionistas (estimados aplicando Índices de Gini) son muy reducidos, tanto entre las mujeres que han recibido el complemento como entre las altas en 2016, y se produce un pequeño empeoramiento de la desigualdad para el total de las pensionistas, ya que el CDM se aplica solo sobre las nuevas pensiones, que son de importe medio superior.

Para reducir la brecha de género en las pensiones en el marco de las políticas de igualdad, se proponen como medidas en el sistema de pensiones:

a) Sustituir el actual CDM por el reconocimiento de un período de cotización adicional por hijo o por un complemento de cuantía fija, con carácter temporal.

La introducción de medidas más equitativas llevaría a sustituir el actual CDM por correcciones que supongan aumentos decrecientes en la pensión. Podría ser un complemento de cuantía fija, como hace Luxemburgo, o el reconocimiento de mayores carreras de cotización por períodos dedicados a la atención y al cuidado de hijos y dependientes, coincidentes o no con períodos efectivamente cotizados, como se hace en Francia, Alemania o Suecia.

El mecanismo de reconocimiento de un periodo de cotización adicional por hijo, añadido al período de cotización efectivo, supone sumar puntos porcentuales al porcentaje de pensión previo, de lo que resulta incrementos decrecientes de pensiones en la escala de pensiones. Además, si el periodo reconocido por hijo se tiene en cuenta para el cálculo del período de carencia, aumenta el número de mujeres con acceso al sistema contributivo.

Si se quiere que la medida no reproduzca los otros aspectos cuestionados, habría que estudiar cómo implantarla de manera que abarcara las pensiones ya causadas, incluyera a madres de un solo hijo y reconociera mayor valor a los hijos con discapacidad. Además, si se pretende mejorar la progresividad de la medida, habrá que limitar el porcentaje máximo de pensión al 100\% (una vez alcanzado dicho porcentaje, los hijos ya no computan). Al mismo tiempo, para que la medida sea igualitaria habrá que estudiar su aplicación de manera indistinta a madres y a padres, como cuidadores. Y, por último, debería ser una medida temporal para que no genere efectos contrarios a los pretendidos en materia de empleo y desarrollo profesional de las mujeres y de reparto de las responsabilidades familiares. 
b) Mejora de las pensiones no contributivas (PNC)

En la reducción de las diferencias de género, tan importante o más que la medida anterior es mejorar las pensiones de menor importe y reducir el número de mujeres sin pensión, haciendo mayor hincapié en la disminución de la brecha de cobertura y de la brecha de las pensiones estimada a partir de la población total, incluyendo a los no pensionistas (que Tinios et al., 2015, estiman para España en el $52 \%$ en 2011, una de las más altas de la UE).

Hay muchas mujeres que no perciben ningún tipo de pensión propia, porque no han realizado trabajo remunerado, no han acumulado el período de carencia exigido o sobrepasan el límite de rentas para la obtención de la PNC (en el que computan las rentas del cónyuge y de otros familiares). De hecho, la debilidad e insuficiencia de cobertura del sistema no contributivo es una potente motivación en la defensa del mantenimiento de las pensiones de viudedad, aunque se trata de pensiones establecidas en función del vínculo familiar e implantadas como reconocimiento del trabajo doméstico de las mujeres de la era industrial, en un contexto de dependencia económica.

La realidad actual requiere desligar las prestaciones sociales de las relaciones familiares y la reducción de las diferencias de género en las pensiones, considerando a todas las mujeres, por tanto, también a las que no tienen acceso a ninguna pensión. El sistema de pensiones requiere de una ampliación de la cobertura del sistema no contributivo, para que deje de ser un sistema anacrónico y asistencial.

Estas propuestas y la actualización de las estimaciones, en base a los datos de la MCVL de futuros años, son las que queremos sean objeto de nuestros futuros trabajos.

\section{Bibliografía}

BALLESTER, M.A. (2016): "El comprometido complemento de pensiones por maternidad en España y su improbable acomodo a la normativa y jurisprudencia antidiscriminatoria de la Unión Europea". Revista Jurídica de los Derechos Sociales. Lex Social, 6, 1, 72-93.

BANCO MUNDIAL (2017): Banco de datos. Consulta 20 de diciembre de 2017 (http://databank.bancomundial.org/data/reports.aspx?source=2\&type=metadata\&series=SP.DYN.TFRT.IN\# )

BETTIO, F., TINIOS, P. \& BETTI, G. (2013): The Gender Gap in Pensions in the UE. European Commission. Consulta 15 de mayo de 2018. DOI: 10.2839/221006. 
BONELL, R. y DEVESA, E. (2017): "El complemento por maternidad de las pensiones de la Seguridad Social en España: Análisis financiero actuarial basado en la MCVL", Economía Española y Protección Social, IX, 117-157.

CEBRIÁN, I., MORENO, G. y TOHARIA, L. (2011): "La estabilidad laboral y los programas de fomento de la contratación indefinida", Hacienda Pública Española / Revista de Economía Pública, 198: 103-127.

CCOO (2016): Complemento por maternidad en las pensiones contributivas de la Seguridad Social, CCOO de Castilla y León. Junta de Castilla y León. Consulta 15 de mayo de 2018 (http://www. castillayleon.ccoo.es/d0509820814ce9d5725b71bb58dc9fb9000054.pdf).

CEBRIÁN, I. y MORENO, G. (2015): "Tiempo cotizado, ingresos salariales y sus consecuencias para las pensiones: diferencias por género al final de la vida laboral", Cuadernos de Relaciones Laborales, 33, 2, 331-328.

Comisión de Seguimiento y Evaluación de los Acuerdos del Pacto de Toledo (CSEPT) (2015a): Comparecencia del señor secretario de Estado de la Seguridad Social (en relación al Informe sobre el complemento de maternidad). Diario de Sesiones del Congreso de los Diputados. Cortes Generales $n^{\circ}$ 853, sesión $n^{\circ}$ 24, 30/6/2015. Consulta 15 de mayo de 2018 (http://www.congreso.es/ public_oficiales/L10/CONG/DS/CO/DSCD-10-CO-853.PDF).

Comisión de Seguimiento y Evaluación de los Acuerdos del Pacto de Toledo (CSEPT) (2015b): Comparecencia en relación al Informe sobre el Plan integral de apoyo a la familia en la Comisión de seguimiento y evaluación de los acuerdos del Pacto de Toledo. Diario de Sesiones del Congreso de los Diputados. Cortes Generales n 868 , sesión n 26 (extraordinaria), 14/7/2015. Consulta 15 de mayo de 2018 (http://www.congreso.es/public_oficiales/L10/CONG/DS/CO/DSCD-10-CO-868. PDF).

Comisión de Seguimiento y Evaluación de los Acuerdos del Pacto de Toledo (CSEPT) (2017): Comparecencias. Diario de Sesiones del Congreso de los Diputados. Cortes Generales $n^{0} 116$, sesión $n^{0}$ 18, 9/2/2017. Consulta 15 de mayo de 2018 (http://www.congreso.es/public_oficiales/L12/CONG/ DS/CO/DSCD-12-CO-116.PDF).

CONSEJO DE LA UE (2003): Informe conjunto de la Comisión y del Consejo sobre la adecuación y la viabilidad de las pensiones (aprobado por el Consejo de Ministros 3/3/2003 (doc 6527/2/03, REV 2). Consulta 15 de mayo de 2018 (https://ec.europa.eu/employment_social/soc-prot/pensions/2003jpr_es.pdf).

CONSEJO DE LA UE (2011): Conclusiones del Consejo de 7 de marzo de 2011 sobre Pacto Europeo por la lgualdad de Género. 2011-2020). Diario Oficial de la UE. 2011/C 155/02). Consulta 15 de mayo de 2018 (http://eur-lex.europa.eu/LexUriServ/LexUriServ.do?uri=OJ:C:2011:155:0010:0013:ES:PDF). 
CONSEJO DE MINISTROS (2015): Plan integral de apoyo a la familia 2015-2017. Consulta 1 de diciembre de 2018 (https://www.mscbs.gob.es/novedades/docs/PIAF-2015-2017.pdf).

DAVAKI, K. (2016): Differences in men's and women's work, care and leisure time. Policy Department for Citizen's Rights and Constitutional Affairs. European Parliament. Consulta 15 de mayo de 2018 (http://www.europarl.europa.eu/RegData/etudes/STUD/2016/556933/IPOL_STU. 2016)556933_ EN.pdf).

DE LA CORTE, I. (2016): "Cuestiones constitucionales sobre el complemento por maternidad. Un estudio comparado con el Derecho de Unión Europea y sus antecedentes jurisprudenciales". Consulta 15 de mayo de 2018 (http://forelab.com/wp-content/uploads/premios/2016/FINALISTA-2016-Cuestiones-constitucionales-sobre-el-complemento-por-maternidad-Ignacio-de-la-Corte-Cala.pdf).

DE LA FLOR, M.L. (2016): "Reflexiones en torno a la pensión de jubilación desde una óptica de género: el nuevo complemento por maternidad", Revista de derecho social, 76, 107-132.

ERRANDONEA, E. (2016): "El nuevo complemento por maternidad y los factores que repercuten sobre la cuantía de las pensiones contributivas de las mujeres", Revista de derecho de la seguridad social, 7, 75-97.

FRERICKS, P., MAIER, R. \& DE GRAAF, W. (2007): "Male norms and female adjustments: The influence of care credits on gender pension gaps in France and Germany", European Societies, Vol. 10 (1), 97-119, DOI: 10.1080/14616690701348810.

GALLEGO, R. (2016): "El complemento de maternidad: una medida discutible para cerrar la brecha de género de las pensiones", Estudios financieros. Revista de trabajo y seguridad social, 403, 19-54.

KLEVEN, H., LANDAIS, C. \& SØGAARD, J.E. (2018): Children and Gender Inequality: Evidence from Denmark. NBER Working Paper No. 24219, Issued in January 2018. DOI: 10.3386/w24219.

LAZARO, N., MOLTÓ, M.L. y SÁNCHEZ, R. (2004): "Desigualdades de género en el trabajo. La brecha de género en el empleo y la distribución de las tareas de cuidado, CIRIEC-España, Revista de Economía Pública, Social y Cooperativa, 50, 55-75.

Instituto Nacional de la Seguridad Social (INSS) (2017): Informe Estadístico 2016. Consulta 15 de mayo de 2018 (http://www.seg-social.es/wps/portal/wss/internet/EstadisticasPresupuestosEstudios/PublicacionesDocumentacion/47999/3680\#229137).

Ministerio de Empleo y Seguridad Social (MESS) (2015): Informe sobre el complemento de maternidad en las pensiones contributivas. Consulta 15 de mayo de 2018 (http://portal.ugt.org/actualidad/2015/octubre/boletin23/infootros02.pdf).

Ministerio de Empleo y Seguridad Social (MESS) (2016): Informe sobre el desarrollo del Pacto de Toledo 2011-2015. Consulta 15 de mayo de 2018 (http://portal.ugt.org/actualidad/2016/NEG_COL_ NUM_26/otros_03.pdf). 
Ministerio de Empleo y Seguridad Social (MESS) (2017): Seguridad Social. Presupuestos Ejercicio 2017. Informe Económico-Financiero. Vol V. Tomo 1. Consulta 15 de mayo de 2018 (http://www. seg-social.es/prdi00/groups/public/documents/binario/223952.pdf)

Ministerio de Trabajo e Inmigración (MTIN) (2008): Informe sobre el desarrollo del Pacto de Toledo. Ministerio de Trabajo e Inmigración. Consulta 15 de mayo de 2018 (http://www.seg-social.es/ prdi00/groups/public/documents/binario/110963.pdf)

Ministerio de Trabajo e Inmigración (MTIN) (2011): Informe de evaluación y reforma del Pacto de Toledo, Ministerio de Trabajo e Inmigración. Consulta 15 de mayo de 2018 (http://www.seg-social.es/prdi00/groups/public/documents/binario/128563.pdf)

MORAL-ARCE, I., PATXOT, C. y SOUTO, G. (2008): "La sostenibilidad del Sistema de pensiones. Una aproximación a partir de la MCVL", Revista de Economía Aplicada, XVI (E-1), 22-66.

MUÑOZ DE BUSTILLO, R. y PINTO, F. (2018): "La curva salarial a lo largo de la vida laboral en España desde la transición. Un análisis longitudinal por cohortes (1981- 2015)". XXV Encuentro de Economía Pública, Valencia, enero 2018, 1-17. Consulta 15 de mayo de 2018 (https://editorialexpress.com/cgi-bin/conference/download.cgi?db_name=25EEB\&paper_id=93).

PATXOT, C., SOUTO, G. y VILLANUEVA, J. (2009): "Fostering the contributory nature of the Spanish retirement pension system: An arithmetic micro-simulation exercise using the MCVL", Presupuesto y Gasto Público, 57, 7-33.

PAZOS, M. (2011): "Demografía, sostenibilidad e igualdad de género", Papeles de Relaciones ecosociales y cambio global, 113, 71-83.

PAZOS, M. (2013): Desigualdades por ley. Las políticas públicas contra la igualdad de género, Los Libros de la Catarata, Madrid.

PÉREZ-SALAMERO, J.M., REGÚLEZ, M. y VIDAL, C. (2016): "Análisis de la representatividad de la MCVL: el caso de las prestaciones del sistema público de pensiones", Hacienda Pública Española / Revista de Economía Pública, 217: 67/130. DOI: 10.7866/HPE-RPE.16.2.3.

RODRÍGUEZ, I.A. (2016): "El nuevo complemento por maternidad en las pensiones contributivas del sistema de la Seguridad Social: puntos críticos (I) y (II)". Trabajo y Derecho: nueva revista de actualidad y relaciones laborales, 16, 1-9 y 17, 1-9.

SALVADOR, C. (2018): Economía de la Seguridad Social. Parte II, Valencia: Tirant Lo Blanch.

TINIOS, P., BETTIO, F., BETTI, G. \& GEORGIADIS, T. (2015): Men, Women and Pensions, European Commission. DOI: 10.2838/200295.

VARA, M.J. (2011): "La desigualdad de género en el sistema público de pensiones: el caso de España". ICE. Tribuna de Economía, 859. Consulta 15 de mayo de 2018 (http://www.revistasice.com/ CachePDF/ICE_859_119-140_885443446B20EB322505DDB2798BB7B6.pdf). 